\title{
Using Antibiograms to Improve Antibiotic Prescribing in Skilled Nursing Facilities
}

\author{
Jon P. Furuno, PhD; $;^{1}$ Angela C. Comer, MPH; $;{ }^{2,3}$ J. Kristie Johnson, PhD, D(ABMM); ${ }^{2,4}$ Joseph H. Rosenberg, BS; $;^{2}$ \\ Susan L. Moore, PhD, MSPH; $;^{5}$ Thomas D. MacKenzie, MD, MSPH; ${ }^{5}$ Kendall K. Hall, MD, MS; ${ }^{6}$ \\ Jon Mark Hirshon, MD, MPH, $\mathrm{PhD}^{2,3,7}$
}

\begin{abstract}
BACKGROUND. Antibiograms have effectively improved antibiotic prescribing in acute-care settings; however, their effectiveness in skilled nursing facilities (SNFs) is currently unknown.

ов IEctive. To develop SNF-specific antibiograms and identify opportunities to improve antibiotic prescribing.

DESIGN AND SETTING. Cross-sectional and pretest-posttest study among residents of 3 Maryland SNFs.

METHODS. Antibiograms were created using clinical culture data from a 6-month period in each SNF. We also used admission clinical culture data from the acute care facility primarily associated with each SNF for transferred residents. We manually collected all data from medical charts, and antibiograms were created using WHONET software. We then used a pretest-posttest study to evaluate the effectiveness of an antibiogram on changing antibiotic prescribing practices in a single SNF. Appropriate empirical antibiotic therapy was defined as an empirical antibiotic choice that sufficiently covered the infecting organism, considering antibiotic susceptibilities.

RESULTS. We reviewed 839 patient charts from SNF and acute care facilities. During the initial assessment period, $85 \%$ of initial antibiotic use in the SNFs was empirical, and thus only $15 \%$ of initial antibiotics were based on culture results. Fluoroquinolones were the most frequently used empirical antibiotics, accounting for $54.5 \%$ of initial prescribing instances. Among patients with available culture data, only $35 \%$ of empirical antibiotic prescribing was determined to be appropriate. In the single SNF in which we evaluated antibiogram effectiveness, prevalence of appropriate antibiotic prescribing increased from $32 \%$ to $45 \%$ after antibiogram implementation; however, this was not statistically significant $(P=.32)$.
\end{abstract}

CONCLUSIONS. Implementation of antibiograms may be effective in improving empirical antibiotic prescribing in SNFs.

Infect Control Hosp Epidemiol 2014;35(S3):S56-S61

Infection is prevalent among residents of skilled nursing facilities (SNFs) due to age-associated decreases in host resistance leading to frequent antibiotic and healthcare exposures. ${ }^{1}$ A recent point prevalence study suggested that approximately $12 \%$ of residents in a nationally representative sample of nursing homes had an infection. ${ }^{1}$ Concerns regarding infections in this population include both infection-associated morbidity and mortality and, additionally, the potential loss of function and illness associated with transfer to an acute-care facility. ${ }^{2,3}$

Despite the high prevalence of infection in SNFs and potential associated poor health outcomes, resources are often limited for diagnosis of infections and to guide empirical antibiotic therapy. ${ }^{4,5} \mathrm{~A}$ recent review by Stone et $\mathrm{al}^{6}$ updating the McGeer criteria for infections in long-term care should assist clinicians in appropriately identifying infections and subsequent appropriate antibiotic prescribing. However, most definitions of infections still require laboratory confirmation, a resource that many SNFs lack or use infrequently. Furthermore, a recent systematic review only identified 4 trials of interventions to reduce potentially inappropriate antibiotic prescribing in long-term care. ${ }^{7}$

In this study, we describe the design and implementation of SNF-specific antibiograms to improve empirical antibiotic prescribing. We estimated the frequency of inappropriate empirical antibiotic prescribing in 3 Maryland SNFs and measured the effect of the antibiograms on antibiotic prescribing in 1 of the SNFs. These data are essential to improve our

Affiliations: 1. Department of Pharmacy Practice, Oregon State University/Oregon Health and Science University College of Pharmacy, Portland, Oregon; 2. Department of Epidemiology and Public Health, University of Maryland School of Medicine, Baltimore, Maryland; 3. National Study Center for Trauma and EMS, University of Maryland School of Medicine, Baltimore, Maryland; 4. Department of Pathology, University of Maryland School of Medicine, Baltimore, Maryland; 5. Department of Patient Safety and Quality, Denver Health and Hospital Authority, Denver, Colorado; and Department of Medicine, University of Colorado Health Sciences Center, Denver Colorado; 6. Center for Quality Improvement and Patient Safety, Agency for Healthcare Research and Quality, Rockville, Maryland; 7. Department of Emergency Medicine, University of Maryland School of Medicine, Baltimore, Maryland.

Received April 2, 2014; accepted April 8, 2014; electronically published September 15, 2014.

(C) 2014 by The Society for Healthcare Epidemiology of America. All rights reserved. 0899-823X/2014/35S3-0009\$15.00. DOI: 10.1086/677818 
understanding of the burden of inappropriate empirical antibiotic prescribing in SNFs and to identify opportunities to improve antibiotic prescribing in this setting.

\section{METHODS}

The methods of this study have been described elsewhere. ${ }^{8}$ In brief, the study was divided into 2 parts. We first performed a needs assessment and cross-sectional study in 3 SNFs and the acute care facilities primarily receiving medically related transfers from the SNFs to develop SNF-specific antibiograms. We then used a pretest-posttest quasi-experimental study to evaluate the effectiveness of the antibiogram to change empirical antibiotic prescribing in 1 of the SNFs. Before study commencement, this study was approved by the institutional review board (IRB) of the University of Maryland, Baltimore (UMB). In addition, the study was approved by facility-specific IRBs at participating facilities where appropriate; others ceded oversight to the UMB IRB.

SNFs were selected and invited to participate on the basis of previous collaboration with the Maryland Long-Term Care Project, which represented different SNF populations and settings in Maryland. ${ }^{9}$ Participating SNFs included a rural, 118 bed, community, for-profit facility with a dedicated dementia floor; an urban, 147-bed, hospital-affiliated, not-for-profit facility; and a 167-bed suburban, for-profit facility.

\section{Data Collection}

Needs assessments consisted of semistructured interviews with the infection/quality control nurses at each participating SNF. All interview participants provided informed consent before participation. Topics discussed during the needs assessments included policies and procedures for microbiological culturing for suspected infections, submitting cultures for analysis, receiving culture and antibiotic susceptibility data, and antibiotic prescribing.

Two authors (A.C.C. and J.H.R.) collected clinical and demographic data on SNF residents from the medical records of each SNF. All data were collected using manual chart review using a standardized form and entered into a Microsoft Access 2007 database. All residents' records were included in the data collection if they resided in the SNF on the day chart review began or resided in the SNF within the previous 6 months and either had a clinical culture collected or were transferred to an acute care hospital. Data collected included demographic characteristics (eg, age and sex), primary and comorbid diagnoses, history and characteristics of infections, culture data (eg, date, sites, bacterial identification, and antibiotic susceptibilities), antibiotic exposures, indwelling medical devices, and history of acute care hospitalization. We defined suspected infections as times when cultures were ordered, doctor's notes were made, and/or antibiotics were started. In addition to SNF data, we collected admission clinical culture (defined as within 48 hours of admission) data from the acute care facility to which the majority of SNF residents were transferred for acute care medical indications during the 6-month period. Admission acute care data were collected to further inform the antibiograms, because organisms represented in the resulting culture data likely originated in the referring SNF.

\section{Development and Implementation of SNF-Specific Antibiograms}

Antibiograms were created by uploading clinical culture data into WHONET, a free, Windows-based software program created by the World Health Organization (WHO) Collaborating Centre for Surveillance of Antimicrobial Resistance. ${ }^{10}$ Antibiogram results were manually entered into a template matrix for ease of interpretation. Consistent with Clinical Laboratory Standards Institute (CLSI) guidelines, only data from the first organism-specific positive culture per resident in the study period was included. ${ }^{11}$ In addition, we had initially planned to create separate antibiograms for each SNF and acute care facility as well as combined antibiograms for SNF and associated acute care facility; however, as the data will reflect, there were not sufficient acute care culture data from 2 of the 3 associated acute care facilities.

The SNF-specific antibiograms were implemented in partnership with the infection/quality control nurses, SNF administrators, and SNF medical directors. The implementation involved multiple in-service presentations at each SNF to present the antibiogram and to explain how to use to use the tool when making antibiotic therapy decisions. Each SNF provided input on the format and structure of their individual antibiogram as well as how it would be implemented (eg, distributed to physicians and nursing staff, posted at nursing stations, and attached to transfer documentation).

\section{Evaluation of Antibiogram Implementation to Improve Empirical Antibiotic Prescribing}

We calculated the proportion of empirical antibiotic prescribing that was determined to be appropriate among residents who received empirical antibiotics and had clinical cultures collected. Appropriate empirical antibiotic therapy was defined as an empirical antibiotic choice that sufficiently covered the infecting organism considering antibiotic susceptibilities. In addition, we evaluated the effectiveness of antibiogram implementation to improve empirical antibiotic prescribing in 1 SNF, the 118-bed, rural facility described previously. We used a quasiexperimental pretest-posttest design and collected resident data for a second 6-month period after implementation. These data were collected on the same data elements as in the initial assessment period using manual chart review by the same co-authors. We then calculated the proportion of empirical antibiotic therapy that was determined to be appropriate in the posttest period. A follow-up survey was developed to assess prescriber exposure to the antibiogram as well as to gather suggestions for changes to 
the tool. As discussed in our previous publication, no prescribers from the SNF responded to the survey. ${ }^{8}$

\section{RESULTS}

The needs assessments provided data on the infrastructure for development and implementation of the antibiograms and identified many similarities across the 3 SNFs. In all facilities, urine cultures were the most frequently ordered clinical cultures, and collection of cultures from other body sites was rare. Wound culturing was not standard practice in these facilities. Two of the 3 SNFs used a clinical microbiology laboratory at an acute care hospital to process their cultures, and the third SNF used a private laboratory on weekdays and contracted with a hospital laboratory on the weekends. All SNFs received culture results via fax from the associated laboratories, which were then communicated to the medical director or residents' physician and placed into the residents' charts.

In total, we reviewed 839 SNF and acute care resident charts to inform the development of the antibiograms. Mean ( \pm standard deviation) patient age in the 3 SNFs ranged from $71.0 \pm 12.6$ years to $82.5 \pm 9.8$ years, the proportion of female residents ranged from $58.9 \%$ to $76.4 \%$, and the proportion of black residents ranged from $10.7 \%$ to $53.3 \%$. The proportions of acute care transfers to a primary-associated hospital were $48 \%, 79 \%$, and $98 \%$.

Table 1 describes the frequency of clinical cultures collected by site over the 6-month initial assessment periods in the 3 participating SNFs. As the needs assessments suggested, urine was the most frequent culture site, accounting for approximately $77.8 \%$ of cultures across all SNFs and as many as $89.4 \%$ of cultures in 1 SNF. Stool cultures were the second most frequent culture site at $14.0 \%$ of all clinical cultures, followed by skin and soft-tissue cultures, which also included wounds at $4.6 \%$. Cultures were ordered in $50 \%$ of suspected infections to confirm diagnoses and empirical therapy choice.

Figure 1 is a composite antibiogram providing susceptibility data for the 3 SNFs. In all SNFs, resistance to cipro- floxacin and trimethoprim-sulfamethoxazole among the Escherichia coli isolates was high; however, resistance to other agents among the different organisms varied by facility. For example, $31 \%$ and $53 \%$ of Proteus mirabilis isolates in SNFs 1 and 2, respectively, were susceptible to ciprofloxacin, but susceptibility was $70 \%$ in SNF 3.

Table 2 displays the frequency of different antibiotic classes used as empirical therapy in the initial assessment period in the 3 participating SNFs. Empirical therapy accounted for $85 \%$ of initial antibiotics prescribed during this period, and as expected, fluoroquinolones were the most frequent antibiotic class prescribed, accounting for $39.8 \%$ of all empirical antibiotics.

Across all SNFs, among residents who received empirical antibiotic therapy and had clinical cultures collected, only $35 \%$ (range, $27.3 \%-38.9 \%$ ) of antibiotic prescribing was determined to be appropriate during the initial assessment period. In the 1 SNF in which we evaluated antibiogram effectiveness, the prevalence of appropriate empirical antibiotic prescribing increased from $32 \%$ to $45 \%$ after implementation of the antibiogram; however, this was not statistically significant $(P=.32)$.

Educational in-services were conducted at each SNF to explain the results of the initial chart reviews and to introduce the antibiograms. In the 1 SNF in which we evaluated the antibiogram, study staff conducted 2 in-services, the first with SNF nursing staff and administrators and the second with SNF nurse managers and physicians. The antibiograms were distributed at these meetings; however, any further exposure to the tool by prescribers could not be assessed given the lack of responses to our follow-up survey.

\section{DISCUSSION}

In this study, we identified and described opportunities to improve empirical antibiotic prescribing in SNFs. As expected, antibiotic therapy was predominately empirical, and rarely were cultures collected to confirm antibiotic choice. During the initial assessment period, $85 \%$ of initial antibiotics

TABLE 1. Frequency of Clinical Cultures in 3 Maryland Skilled Nursing Facilities (SNFs) by Site over a 6-Month Period

\begin{tabular}{lccccc}
\hline & \multicolumn{5}{c}{ No. (\%) of cultures } \\
\cline { 2 - 5 } Culture site & \multicolumn{2}{c}{ SNF 1 } & & & \\
\cline { 2 - 5 } & Pretest & Posttest & SNF 2 & SNF 3 & Total \\
\hline Urine & $75(78.1)$ & $54(75.0)$ & $155(73.5)$ & $76(89.4)$ & $306(78.1)$ \\
Blood & $5(5.2)$ & $2(2.8)$ & $3(1.4)$ & $0(0)$ & $8(2.0)$ \\
Skin/soft tissue (includes wounds) & $2(2.1)$ & $4(5.6)$ & $16(7.6)$ & $0(0)$ & $18(4.6)$ \\
Stool & $12(12.5)$ & $8(11.1)$ & $35(16.6)$ & $8(9.4)$ & $55(14.0)$ \\
Respiratory tract & $1(1.0)$ & $4(5.6)$ & $2(<1.0)$ & $1(1.2)$ & $4(1.0)$ \\
Other & $1(1.0)$ & $0(0.0)$ & $0(0)$ & $0(0)$ & $1(<1.0)$ \\
$\quad$ Total & $96(100)$ & $72(100)$ & $211(100)$ & $85(100)$ & $392(100)$ \\
\hline
\end{tabular}

NOTE. Both pretest and posttest data are provided for SNF 1. Only pretest data were available for SNFs 2 and 3. 


\begin{tabular}{|c|c|c|c|c|c|c|c|c|c|c|c|c|c|c|c|c|c|c|c|c|c|c|c|}
\hline & & & \multicolumn{3}{|c|}{ Aminoglycosides } & \multicolumn{3}{|c|}{ B-Lactams } & \multicolumn{3}{|c|}{ Carbapenems } & \multicolumn{5}{|c|}{ Cephalosporins } & \multicolumn{3}{|c|}{ Quinolones } & \multicolumn{4}{|c|}{ Others } \\
\hline Gram (-) & SNF & $\begin{array}{l}\text { \# of } \\
\text { isolates }\end{array}$ & 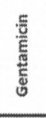 & 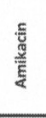 & 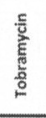 & $\frac{\underline{\underline{E}}}{\frac{5}{\bar{c}}}$ & 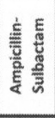 & 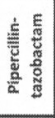 & 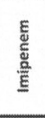 & 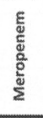 & $\begin{array}{l}\text { E } \\
\frac{\mathrm{E}}{\mathrm{E}} \\
\frac{\mathrm{g}}{\mathrm{E}} \\
\mathrm{E}\end{array}$ & 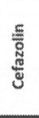 & 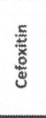 & 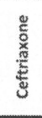 & 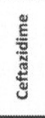 & 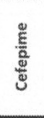 & 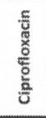 & 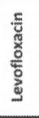 & 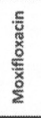 & 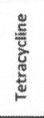 & 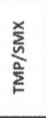 & 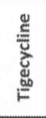 & 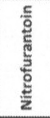 \\
\hline \multirow{3}{*}{ Escherichia coli } & 1 & 13 & 85 & 100 & 92 & 39 & & 92 & 100 & & & 85 & 100 & 100 & 100 & & 39 & & & & 39 & & 92 \\
\hline & 2 & 19 & 84 & - & & 32 & 42 & 100 & 100 & & 100 & 84 & & & & & 37 & 37 & & 58 & 58 & & \\
\hline & 3 & 16 & 69 & 100 & 71 & 31 & & 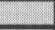 & 100 & 100 & 100 & 88 & & 93 & & 93 & 38 & - & 36 & 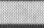 & 50 & 100 & 94 \\
\hline \multirow{3}{*}{ Klebsiella sp } & 1 & 7 & 57 & 71 & 71 & & & 86 & 86 & & 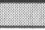 & 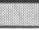 & & 57 & 57 & & 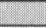 & & - & & 71 & 紧 & 29 \\
\hline & 2 & 9 & 100 & -1 & ב- & 0 & 67 & 89 & & & 89 & 56 & 2 & 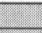 & 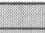 & & 67 & 78 & & 78 & 89 & & W \\
\hline & 3 & 0 & & & 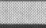 & & & & & & 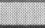 & & & $\overline{2}$ & 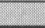 & & 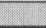 & 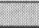 & & 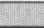 & . & & \\
\hline \multirow{3}{*}{ Proteus mirabilis } & 1 & 13 & 83 & 100 & 92 & 92 & & 100 & & & & & 82 & 100 & 100 & & 31 & & & 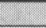 & 69 & & \\
\hline & 2 & 17 & 100 & & & 82 & & 94 & & & 100 & 82 & & & & & 53 & 59 & & 0 & 88 & & \\
\hline & 3 & 10 & 100 & 100 & 100 & 60 & & & & 100 & 100 & 70 & & 70 & & 70 & 70 & & 50 & & 70 & & 0 \\
\hline \multirow{3}{*}{$\begin{array}{l}\text { Pseudomonas } \\
\text { aeruginosa }\end{array}$} & 1 & 4 & 100 & 100 & 100 & & & 100 & 100 & & & & & & 100 & & 75 & & & & . & & \\
\hline & 2 & 0 & & & & & & & & & & 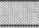 & & 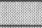 & & & 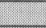 & & & & 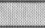 & & \\
\hline & 3 & 0 & & & & & & & & & & & & & & & $8 x$ & & & & & & S \\
\hline & & & \multicolumn{4}{|c|}{ Penicillins } & Tetrac & clines & \multicolumn{3}{|c|}{ Quinolones } & \multicolumn{12}{|c|}{ Others } \\
\hline $\operatorname{Gram}(+)$ & SNF & $\begin{array}{l}\text { \# of } \\
\text { Isolates }\end{array}$ & 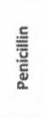 & 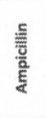 & 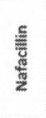 & 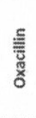 & 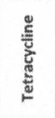 & 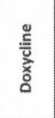 & 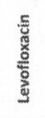 & 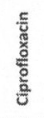 & 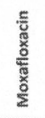 & 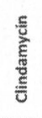 & 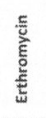 & 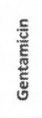 & 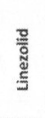 & 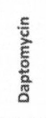 & 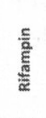 & 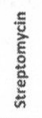 & & & 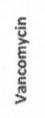 & 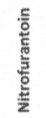 & 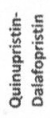 \\
\hline \multirow{3}{*}{$\begin{array}{c}\text { Staphylococcus } \\
\text { aureus }\end{array}$} & 1 & 8 & 0 & & 0 & 0 & 100 & & & 0 & 0 & & & 88 & 100 & & 100 & & & & 100 & 100 & \\
\hline & 2 & 11 & 0 & & & 27 & 70 & 100 & & & & 20 & 20 & 80 & 100 & & 100 & & & & 100 & & 100 \\
\hline & 3 & 0 & & & & & & & & & & & & & & & & & & & & & \\
\hline \multirow{3}{*}{ Enterococcus sp } & 1 & 4 & 100 & 100 & & & 25 & & & & & & & & & & & & & & 100 & 100 & \\
\hline & 2 & 11 & & 73 & & & & & 57 & & & & & 90 & & 100 & & 82 & & & 64 & & \\
\hline & 3 & 0 & $=$ & & & & & & & & & & & & & & & & & & & & \\
\hline \multirow{3}{*}{ Streptococcus sp } & 1 & A & 100 & 100 & & & 25 & & & & & & & & & & & & & & 100 & & \\
\hline & 2 & 0 & & & & & & & & & & & & & & & & & & & & 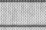 & \\
\hline & 3 & 0 & & & & & & & & & & & & & & & & & & & & & \\
\hline
\end{tabular}

FIgURE 1. Composite antibiogram for 3 Maryland skilled nursing facilities (SNFs). Each cell provides the proportion of each organism susceptible to the associated antibiotic. TMP/SMX, trimethoprim-sulfamethoxazole.

prescribed were prescribed empirically. Fluoroquinolones were the most frequently used empirical antibiotics, accounting for $40 \%$ of initial prescribing instances, and this prevalence remained high following antibiogram implementation in the 1 SNF (39\%) in which we evaluated antibiogram effectiveness, despite the antibiogram suggesting that $66 \%$ of gram-negative organisms tested against fluoroquinolones were resistant to this drug class. Among patients with culture data available, only $35 \%$ of empirical antibiotic prescribing was determined to be appropriate. Prevalence of appropriate antibiotic prescribing in the evaluated SNF increased from $32 \%$ to $45 \%$ after implementation of the antibiogram. These data suggest that implementation of antibiograms may be effective in improving empirical antibiotic prescribing in SNFs; however, there are several challenges that must be overcome for this to be the case.

Despite the challenges faced by SNFs and other residential care facilities with respect to diagnosis and treatment of infections, there are relatively few published studies describing interventions to improve these practices. As we described earlier, a recent systematic review only identified 4 trials of interventions to improve antibiotic prescribing in long-term care. ${ }^{7}$ The 4 included studies were all cluster randomized trials, and all used educational materials aimed at either physicians only or physicians and nurses. ${ }^{12-15}$ Two studies specifically aimed to improve prescribing for urinary tract infections (UTIs), 1 study specifically focused on nursing home-acquired pneumonia, and 1 study focused on antibi- otic prescribing for a variety of infections that included UTIs, lower respiratory tract infections, skin and soft-tissue infections, and septicemia of unknown origin. ${ }^{13}$ Although these were all well-designed and important studies, they are not directly comparable to our study. The studies included in the review primarily aimed to reduce antibiotic use or improve the proportion of prescribing that complied with guidelines developed by the investigators rather than to improve antibiotic prescribing using local antibiotic susceptibility data. However, these studies did indicate that educational materials and feedback can invoke changes in prescribing behavior, and all studies observed at least some decrease in antibiotic use or an increase in the proportion of antibiotic use that was consistent with their guidelines or treatment algorithm.

Our observation that the vast majority of antibiotic prescribing was empirical supports previous findings and reinforces the need for tools like antibiograms to guide these practices. In addition, cultures were ordered in only $50 \%$ of suspected infections to confirm diagnoses and empirical therapy choice, and when they were collected, they often suggested that the chosen therapy was inappropriate (ie, insufficient to treat the infecting organism, given antibiotic susceptibilities). The implementation of the antibiogram in 1 SNF did suggest a reduction in inappropriate therapy, but this was not found to be statistically significant, possibly as a result of the relatively short follow-up period exacerbated by a continued low frequency of culturing.

Although development and implementation of antibio- 
TABLE 2. Frequency of Initial Empirical Antibiotic Choice by Class in 3 Maryland Skilled Nursing Facilities (SNFs)

\begin{tabular}{|c|c|c|c|c|c|}
\hline \multirow[b]{3}{*}{ Antibiotic class } & \multicolumn{5}{|c|}{ No. (\%) of cultures } \\
\hline & \multicolumn{2}{|c|}{ SNF 1} & \multirow[b]{2}{*}{ SNF 2} & \multirow[b]{2}{*}{ SNF 3} & \multirow[b]{2}{*}{ Total } \\
\hline & Pretest & Posttest & & & \\
\hline Aminoglycosides & $9(9.5)$ & $1(1.1)$ & $2(1.6)$ & $8(9.2)$ & $20(5.1)$ \\
\hline Carbapenems & $0(0)$ & $0(0)$ & $0(0)$ & $0(0)$ & $0(0)$ \\
\hline Cephalosporins & $12(12.6)$ & $19(21.6)$ & $13(10.7)$ & $17(19.5)$ & $61(15.6)$ \\
\hline Fluoroquinolones & $39(41.1)$ & $34(38.6)$ & $57(46.7)$ & $26(29.9)$ & $156(39.8)$ \\
\hline Vancomycin & $2(2.1)$ & $0(0)$ & $3(2.5)$ & $2(2.3)$ & $7(1.8)$ \\
\hline Trimethoprim-sulfamethoxazole & $5(5.3)$ & $6(6.8)$ & $10(8.2)$ & $10(11.5)$ & $31(7.9)$ \\
\hline Tetracyclines & $1(1.1)$ & $2(2.3)$ & $8(6.6)$ & $2(2.3)$ & $13(3.3)$ \\
\hline Nitrofurantonin & $1(1.1)$ & $4(4.5)$ & $0(0)$ & $1(1.1)$ & $6(1.5)$ \\
\hline Metronidazole & $8(8.4)$ & $6(6.8)$ & $12(9.8)$ & $1(1.1)$ & $27(6.9)$ \\
\hline Other & $18(18.9)$ & $16(18.2)$ & $17(13.9)$ & $20(23.0)$ & $71(18.1)$ \\
\hline
\end{tabular}

NOTE. Note that both pretest and posttest data are provided for SNF 1. Only pretest data were available for SNFs 2 and 3.

grams is a necessary component of improving the effectiveness of empirical antibiotic prescribing, this alone will not be sufficient. Incorporating the knowledge into common practice and changing the healthcare provider culture in SNFs is essential toward improving prescribing behavior and sustaining the effect of interventions over time. Of the previously described studies, only Monette et al $^{13}$ assessed whether the effects of the intervention persisted over time. Three to 6 months after the intervention, experimental facilities still had a higher frequency of antibiotic prescribing that adhered to the recommended guidelines compared with control facilities, but this effect was no longer statistically significant. The educational in-services conducted with each SNF to implement the antibiogram likely had an impact on the infection control knowledge of the SNF prescribers; however, we believe the antibiograms themselves played a larger role. The in-services were single interactions between the study staff and the SNF clinical and administrative staff. In the 1 SNF with followup data, 6 months lapsed between the in-service and when the follow-up data were collected. The antibiogram for this facility was pocket-sized to travel with nurses and physicians, and it was photocopied to the back of the transfer forms as well. Although we do not have the data to verify that SNF prescribers in this facility were exposed more often to the antibiogram itself than the initial in-service, we suspect this was the case and feel comfortable in attributing the improvements in antibiotic prescribing seen at the SNF to the antibiogram. Unfortunately, the lack of SNF prescriber responses to our follow-up survey was a challenge and limitation to our findings. ${ }^{8}$

As noted previously, there are a number of challenges and barriers toward development and implementation of antibiograms in SNFs. ${ }^{8}$ The infrequency of culturing may be due to a lack of education regarding when to culture as well the associated costs, and lack of laboratory resources and dedicated personnel. A previous study of infection control resources in Maryland suggested that long-term care facilities reported an average of 0.3 full-time equivalent infection control professionals per 200 beds. ${ }^{16}$ In addition to those challenges, our study was limited by a number of factors. The research was conducted in 3 SNFs in Maryland, and the results may not be generalizable to other settings. In addition, because we limited data collection for residents who resided in the SNFs within the previous 6 months to those who had clinical culture collected or a transfer to an acute care facility, we may have missed residents with infections that did not have these exposures. Furthermore, our definition of appropriate antibiotic use was based solely on antibiotic susceptibilities of the cultured organism and did not include other important considerations, such as dose and duration, drugdrug interactions, suspected infection site, or liver and renal function. Finally, with respect to the evaluation of effectiveness of antibiogram implementation to improve empirical antibiotic prescribing, we were only able to study this in one of the SNFs, and there are well-described limitations of single site, pretest-posttest studies that should be taken into account when viewing our results. ${ }^{17-19}$

In summary, at present there are limited data on the use of antibiograms or other interventions to improve empirical antibiotic prescribing in SNFs. However, because there is a substantial at-risk population in these facilities, improving antibiotic prescribing practices is important to decrease potential morbidity, mortality, and costs and to improve the quality of life for the SNF residents. Thus, future research should build on these results through further evaluation of antibiograms in combination with educational outreach and support to improve antibiotic prescribing in SNFs.

\section{ACKNOWLEDGMENTS}

We thank Nimalie Stone, MD, who served as Technical Advisor on this project, for her thoughtful input throughout the course of this study.

Financial support. This project was supported under contract number 
HHSA290200600020I, ACTION Task Order 9, "Using Nursing Home Antibiograms to Improve Antibiotic Prescribing and Delivery," from the Agency for Healthcare Research and Quality (AHRQ), US Department of Health and Human Services. The findings and conclusions in this document are those of the authors, who are responsible for its content, and do not necessarily represent the views of AHRQ. No statement in this report should be construed as an official position of AHRQ or of the US Department of Health and Human Services.

Potential conflicts of interest. J.P.F, and J.K.J. report having received research support from Cubist Pharmaceuticals. J.K.J. reports having received research support from Nanosphere. All other authors report no conflicts of interest relevant to this article. All authors submitted the ICMIE Form for Disclosure of Potential Conflicts of Interest, and the conflicts that the editors consider relevant to this article are disclosed here.

Address correspondence to Jon P. Furuno, PhD, OSU/OHSU College of Pharmacy, Department of Pharmacy Practice, 3303 SW Bond Avenue, Mail Code: CH12C, Portland, OR 97239 (furuno@ohsu.edu).

Presented in part: 51st Annual Interscience Conference on Antimicrobial Agents and Chemotherapy; Chicago IL; September 2011.

\section{REFERENCES}

1. Dwyer LL, Harris-Kojetin LD, Valverde RH, et al. Infections in long-term care populations in the United States. J Am Geriatr Soc 2013;61(3):342-349.

2. Strausbaugh LJ, Joseph CL. The burden of infection in longterm care. Infect Control Hosp Epidemiol 2000;21(10):674-679.

3. Boockvar KS, Gruber-Baldini AL, Burton L, Zimmerman S, May $\mathrm{C}$, Magaziner J. Outcomes of infection in nursing home residents with and without early hospital transfer. J Am Geriatr Soc 2005; 53(4):590-596.

4. Smith PW, Bennett G, Bradley S, et al. SHEA/APIC guideline: infection prevention and control in the long-term care facility, July 2008. Infect Control Hosp Epidemiol 2008;29(9):785-814.

5. Nicolle LE. Antimicrobial stewardship in long term care facilities: what is effective? Antimicrob Resist Infect Control 2014;3(1): 6-2994-3-6.

6. Stone ND, Ashraf MS, Calder J, et al. Surveillance definitions of infections in long-term care facilities: revisiting the McGeer criteria. Infect Control Hosp Epidemiol 2012;33(10):965-977.

7. Fleming A, Browne J, Byrne S. The effect of interventions to reduce potentially inappropriate antibiotic prescribing in longterm care facilities: a systematic review of randomised controlled trials. Drugs Aging 2013;30(6):401-408.

8. Hirshon JM, Comer AC, Rosenberg JH, et al. Methodological challenges associated with developing and implementing antibiograms in nursing homes. In: Battles, JB, Cleeman JI,
Kahn KL, Weinberg DA, eds. Advances in the Prevention and Control of HAIs. Rockville, MD: Agency for Healthcare Research and Quality, 2014. http://www.ahrq.gov/professionals /quality-patient-safety/patient-safety-resources/resources /advances-in-hai/hai-article9.html. Accessed August 22, 2014.

9. University of Maryland Baltimore Gerontology Programs. Maryland long-term care project. http://gerontology.umaryland.edu /longterm.html. Updated 2013. Accessed August 22, 2014.

10. World Health Organization (WHO). WHONET software. http://www.who.int/drugresistance/whonetsoftware/en/. Updated 2011. Accessed August 22, 2014.

11. Clinical Laboratory Standards Institute (CLSI). Performance standards for antimicrobial susceptibility testing; twenty-first informational supplement. 2011. CLSI document M100-S21.

12. Loeb M, Brazil K, Lohfeld $L$, et al. Effect of a multifaceted intervention on number of antimicrobial prescriptions for suspected urinary tract infections in residents of nursing homes: cluster randomised controlled trial. BMJ 2005;331(7518):669.

13. Monette J, Miller MA, Monette $M$, et al. Effect of an educational intervention on optimizing antibiotic prescribing in long-term care facilities. J Am Geriatr Soc 2007;55(8):1231-1235.

14. Pettersson E, Vernby A, Molstad S, Lundborg CS. Can a multifaceted educational intervention targeting both nurses and physicians change the prescribing of antibiotics to nursing home residents? a cluster randomized controlled trial. J Antimicrob Chemother 2011;66(11):2659-2666.

15. Naughton BJ, Mylotte JM, Ramadan F, Karuza J, Priore RL. Antibiotic use, hospital admissions, and mortality before and after implementing guidelines for nursing home-acquired pneumonia. J Am Geriatr Soc 2001;49(8):1020-1024.

16. Roup BJ, Roche JC, Pass M. Infection control program disparities between acute and long-term care facilities in Maryland. Am J Infect Control 2006;34(3):122-127.

17. Harris AD, Bradham DD, Baumgarten M, Zuckerman IH, Fink JC, Perencevich EN. The use and interpretation of quasiexperimental studies in infectious diseases. Clin Infect Dis 2004; 38(11):1586-1591.

18. Harris AD, Lautenbach E, Perencevich E. A systematic review of quasi-experimental study designs in the fields of infection control and antibiotic resistance. Clin Infect Dis 2005;41(1):7782.

19. Shardell M, Harris AD, El-Kamary SS, Furuno JP, Miller RR, Perencevich EN. Statistical analysis and application of quasi experiments to antimicrobial resistance intervention studies. Clin Infect Dis 2007;45(7):901-907. 